\title{
ON THE NOTION OF $n$-CARDINALITY
}

\author{
TEODOR C. PRZYMUSIŃSKI
}

\begin{abstract}
In this paper we introduce and investigate the notion of $n$-cardinality, which turned out to be useful in constructions involving product spaces and has a number of interesting applications.
\end{abstract}

In this paper we introduce and investigate the notion of $n$-cardinality, which turned out to be useful in constructions involving product spaces and has a number of interesting applications (see $[\mathbf{P}],\left[\mathbf{P}_{\mathbf{1}}\right],\left[\mathbf{P}_{\mathbf{2}}\right]$, and $\left[\mathbf{P}_{\mathbf{3}}\right]$ ).

The notion of $n$-cardinality arose after discussions with Eric van Douwen, who also proved an important case of Theorem 1 (Corollary 1). The author is grateful to him for his valuable suggestions.

Throughout this paper $n$ denotes a natural number and $c=2^{\omega}$. Let $X$ be an arbitrary set. For a point $p=\left(p_{1}, \ldots, p_{n}\right)$ from $X^{n}$ by $\hat{p}$ we shall denote the set $\left\{p_{1}, \ldots, p_{n}\right\}$ of coordinates of $p$. By $p_{i}$ we shall always mean the $i$ th coordinate of $p$. For undefined notions and symbols the reader is referred to [E].

LEMMA 1. For a subset $A$ of $X^{n}$ the following cardinals are well defined and they are equal provided that one of them or-equivalently-all of them are infinite:

(i) $\max \left\{|B|: B \subset A\right.$ and $p_{i} \neq q_{i}$, for $i=1,2, \ldots, n$ and every two distinct points $p$ and $q$ from $B$ \};

(ii) $\max \{|B|: B \subset A$ and $\hat{p} \cap \hat{q}=\varnothing$, for every two distinct points $p$ and $q$ from $B$ \};

(iii) $\min \left\{|Y|: Y \subset X\right.$ and $\left.A \subset \cup_{i=1}^{n}\left(X^{i-1} \times Y \times X^{n-i}\right)\right\}$.

Proof. Let us denote by $\tau$ the cardinal number defined in (iii). Since $\tau$ is well defined it suffices to show that: (a) if $\tau \geqslant \omega$, then cardinals described in (i) and (ii) coincide with $\tau$ and (b) if $\tau<\omega$, then cardinals described in (i) and (ii) are finite.

Let us note first that if $B$ is a subset of $A$ such that $p_{i} \neq q_{i}$, for $i=1$, $2, \ldots, n$ and every two distinct points $p$ and $q$ from $B$, then $|B| \leqslant n \cdot \tau$. Indeed, let $Y$ be a subset of $X$ of cardinality $\tau$ such that $A \subset \cup_{i=1}^{n}\left(X^{i-1} \times\right.$ $\left.Y \times X^{n-i}\right)$. For every $i=1,2, \ldots, n$ and every $y \in Y$ there exists at most one $p \in B$ such that $p_{i}=y$, therefore $|B| \leqslant n \cdot \tau$. From this fact we deduce (b) and infer that in order to prove (a) it suffices to construct a subset $B$ of $A$

Received by the editors April 26, 1977 and, in revised form, May 24, 1977 and July 11, 1977.

AMS (MOS) subject classifications (1970). Primary 54A25, 54B10, 04A15; Secondary 54E50, $54 \mathrm{D} 20$. 
of cardinality $\tau$ such that $\hat{p} \cap \hat{q}=\varnothing$, for every two distinct points $p, q \in B$.

Assume that $\tau \geqslant \omega$. We shall construct points $p(\alpha)$ of $B$, for $\alpha<\tau$, by transfinite recursion. Assume that points $p(\beta) \in A$ have been constructed for $\beta<\alpha$ so that $\hat{p}(\beta) \cap \hat{p}(\gamma)=\varnothing$, if $\beta \neq \gamma$.

The set $Z=\cup\{\hat{p}(\beta): \beta<\alpha\}$ has cardinality $<\tau$ and therefore there exists a point

$$
p(\alpha) \in A \backslash \bigcup_{i=1}^{n}\left(X^{i-1} \times Z \times X^{n-i}\right) .
$$

Clearly $\hat{p}(\alpha) \cap \hat{p}(\beta)=\varnothing$, for every $\beta<\alpha$, which completes the proof of the Lemma.

Defintion 1. For a subset $A$ of $X^{n}$, where $X$ is an arbitrary set, we define the $n$-cardinality $|A|_{n}$ of $A$ (with respect to $X^{n}$ ) by $|A|_{n}=\max \{|B|: B \subset A$ and $p_{i} \neq q_{i}$, for every two distinct points $p$ and $q$ from $B$ and $i=1$, $2, \ldots, n\}$. We say that $A$ is $n$-countable (n-uncountable) if $|A|_{n} \leqslant \omega\left(|A|_{n}>\right.$ $\omega)$.

It follows from Lemma 1 that $n$-cardinality is well defined and moreover:

(1) $|A|_{1}=|A|$; i.e. $n$-cardinality generalizes the notion of cardinality;

$$
\begin{gathered}
|A|_{n} \leqslant|A| ; \\
|A|_{2}=\min \{|Y|: A \subset Y \times X \cup X \times Y\}, \\
\text { provided that }|A|_{2} \text { is infinite. }
\end{gathered}
$$

Remark 1 . We can analogously define the $n$-cardinality of a subset $A$ of $\prod_{i=1}^{n} X_{i}$, where $X_{i}$ 's are arbitrary sets, however, this potentially more general definition can be reduced to the previous one by observing that the so defined $n$-cardinality coincides with the $n$-cardinality of $A$ with respect to $X^{n}$, where $X=\bigoplus_{i=1}^{n} X_{i}$. Making use of this observation, one can easily show that all results proved in this paper for subsets of $X^{n}$ are actually valid-after obvious modifications-for subsets of the products $\prod_{i=1}^{n} X_{i}$.

The following theorem generalizes a result of van Douwen (see Corollary 1).

THEOREM 1 (MAIN). Let $X$ be a complete separable metric space and $B$ a Borel subset of $X^{n}$. The following statements are equivalent:

(i) $B$ is $n$-uncountable;

(ii) $B$ has $n$-cardinality continuum;

(iii) B contains a homeomorphic image $h(C)$ of the Cantor set $C$ such that $\hat{h}(x) \cap \hat{h}(y)=\varnothing$, for $x \neq y$;

(iv) B contains a homeomorphic image $h(C)$ of the Cantor set $C$ by the diagonal

$$
h=\bigwedge_{i=1}^{n} h_{i}: C \rightarrow X^{n}
$$

of homeomorphic embeddings $h_{i}: C \rightarrow X$;

(v) (for $n>1) B$ contains the graph of a homeomorphic embedding $h$ : 
$C \rightarrow X^{n-1}$ of a Cantor subset $C$ of $X$ into $X^{n-1}$ such that $\hat{h}(x) \cap \hat{h}(y)=\varnothing$, for $x \neq y$;

(vi) $($ for $n>1) B$ contains the graph of the diagonal $h=\triangle_{i=2}^{n} h_{i}: C \rightarrow X^{n-1}$ of homeomorphic embeddings $h_{i}: C \rightarrow X$ of a Cantor subset $C$ of $X$ into $X$.

Proof. It follows immediately from Lemma 1 that either of the conditions (ii)-(vi) implies (i). We shall show the converse.

It is known that every Borel subset of a separable complete metric space is a continuous image of the space $P$ of irrationals (cf. [K, Theorem 1, Chapter III, §37]). Let $f: P \rightarrow B$ be a continuous mapping of $P$ onto $B$ and assume that $|B|_{n}>\omega$. Let us choose an arbitrary complete metric on $P$. By Lemma 1 there exists a collection $\{p(s)\}_{s \in S}$ of points $B$ such that $\hat{p}(s) \cap \hat{p}\left(s^{\prime}\right)=\varnothing$, for $s \neq s^{\prime}$ and $|S|=\omega_{1}$.

For each $s \in S$ choose an $x_{s} \in f^{-1}(p(s))$ and put $T=\left\{x_{s}\right\}_{s \in S}$. Without loss of generality we can assume that $T$ is dense-in-itself (otherwise, since $T$ is second countable, by the Bernstein Theorem we would remove countably many points from $S$ and $T$ ).

For each $m=1,2, \ldots$ and every sequence $\left(d_{1}, \ldots, d_{m}\right)$, where $d_{i}=0$ or 1, we will define a point $t\left(d_{1}, \ldots, d_{m}\right) \in T$ and a closed ball $B\left(d_{1}, \ldots, d_{m}\right)$ in $P$ with the center at the point $t\left(d_{1}, \ldots, d_{m}\right)$ and radius $<1 / m$ so that:

$$
B\left(d_{1}, \ldots, d_{m}\right) \subset B\left(d_{1}, \ldots, d_{m-1}\right), \text { for } m>1 ;
$$

for each pair $\left(d_{1}, \ldots, d_{m}\right)$ and $\left(d_{1}^{\prime}, \ldots, d_{m}^{\prime}\right)$ of distinct

$(5)_{m} \quad$ sequences there exist disjoint subsets $V_{0}$ and $V_{1}$ of $X$ such that $f\left(B\left(d_{1}, \ldots, d_{m}\right)\right) \subset V_{0}^{n}$ and $f\left(B\left(d_{1}^{\prime}, \ldots, d_{m}^{\prime}\right)\right) \subset V_{1}^{n}$.

Let $m=1$ and choose two distinct points $t(0)$ and $t(1)$ from $T$. Since $X$ is Hausdorff, there exist disjoint open subsets $V_{0}$ and $V_{1}$ of $X$ with $\hat{f}(t(j)) \subset V_{j}$, for $j=0,1$. By the continuity of $f$ there exist closed balls $B(0)$ and $B(1)$ with centers at $t(0)$ and $t(1)$, respectively, and radii $<1$ such that $f(B(j)) \subset V_{j}^{n}$, for $j=0,1$, which completes the first step of the inductive construction.

Assume that $m \geqslant 2$ and that an inductive step has been made for $m-1$. Let us take an arbitrary sequence $\left(d_{1}, \ldots, d_{m-1}\right)$ and find two distinct points $t_{j}=t\left(d_{1}, \ldots, d_{m-1}, j\right), j=0,1$, from $T$ belonging to the interior of $B\left(d_{1}, \ldots, d_{m-1}\right)$. Such points exist because $T$ is dense-in-itself. We can find two disjoint open subsets $V_{j}, j=0,1$, of $X$ such that

$$
\hat{f}\left(t\left(d_{1}, \ldots, d_{m-1}, j\right)\right) \subset V_{j}, \quad j=0,1 .
$$

There exist closed balls $B\left(d_{1}, \ldots, d_{m-1}, j\right), j=0,1$, with centers at the points $t_{j}$ and radii $<1 / m$ such that

$$
B\left(d_{1}, \ldots, d_{m-1}, j\right) \subset B\left(d_{1}, \ldots, d_{m-1}\right)
$$

and

$$
f\left(B\left(d_{1}, \ldots, d_{m-1}, j\right)\right) \subset V_{j}^{n}, \quad j=0,1 .
$$


It is easy to see that the conditions $(4)_{m}$ and $(5)_{m}$ are satisfied, which completes our inductive construction.

One easily sees that the subset

$$
C=\bigcap_{m=1}^{\infty}\left(\bigcup\left\{B\left(d_{1}, \ldots, d_{m}\right):\left(d_{1}, \ldots, d_{m}\right) \in\{0,1\}^{m}\right\}\right)
$$

of $P$ is homeomorphic to the Cantor set (cf. [K, Chapter III, §36,I]) and that the continuous mapping $h=f \mid C: C \rightarrow B \subset X^{n}$ has the property

$$
\hat{h}(x) \cap \hat{h}(y)=\varnothing, \quad \text { for } x \neq y,
$$

in particular, $h$ is one-to-one. As a one-to-one continuous mapping into a Hausdorff space defined on a compact space $C$, the mapping $h$ is a homeomorphic embedding. Therefore, (iii) is satisfied and consequently, by Lemma 1 , also (ii) follows.

Let $h(x)=\left(h_{1}(x), \ldots, h_{n}(x)\right)$, for $x \in C$. Since the mappings $h_{i}$ are continuous and one-to-one, they are homeomorphic embeddings and (iv) holds.

Assume that $n>1$ and let $C^{1}=h_{1}(C) \subset X$ and $h_{i}^{1}=h_{i} \circ h_{1}^{-1}: C^{1} \rightarrow X$, for $i=2,3, \ldots, n$. Clearly $C^{1}$ is homeomorphic to the Cantor set, $h_{i}^{1}$ 's are homeomorphic embeddings of $C^{1} \subset X$ into $X$ and the graph of the diagonal $h^{1}=\triangle_{i=2}^{n} h_{i}^{1}: C^{1} \rightarrow X^{n-1}$ coincides with $h(C)$. This shows that also conditions (v) and (vi) are satisfied and completes the proof.

RemarK 2. It follows from the above proof that conditions (i)-(vi) are actually equivalent for every analytic subset $B$ of $X^{n}$, where $X$ is an arbitrary Hausdorff space (analytic sets are continuous images of irrationals).

Remark 3. R. Pol pointed out that Theorem 1 (and also Theorem 3) can be derived from the results obtained recently by $K$. Kuratowski $\left[K_{1}\right.$, Corollary 3], however, the direct proof of these theorems seems to be simpler.

The following corollary has been first proved by van Douwen [vD].

Corollary 1. Let $X$ be a separable complete metric space. A closed subset $F$ of $X^{n}$ is either $n$-countable or has $n$-cardinality continuum.

Corollary 2. Let $X$ be a separable complete metric space. $A$ Borel subset $B$ of $X^{2}$ is either contained in $(X \times A) \cup(A \times X)$, with $A$ countable, or it contains a graph of a homeomorphic embedding $h: C \rightarrow X$ of a Cantor subset $C$ of $X$ into $X$.

COROLlary 3 (ALEXANDROV-HaUSDORfF). Every uncountable Borel subset of a separable complete metric space contains a Cantor set $C$ and therefore, has cardinality continuum.

The next theorem (and its corollary) generalizes the classical theorem of Bernstein (cf. [K, Theorem 1, §40, I]) on the existence of totally imperfect subsets of the real line and plays an important role in applications of $n$-cardinality (see $[\mathbf{P}],\left[\mathbf{P}_{\mathbf{1}}\right],\left[\mathbf{P}_{\mathbf{2}}\right]$, and $\left[\mathbf{P}_{\mathbf{3}}\right]$ ).

THEOREM 2. Let $X$ be a separable complete metric space. There exist disjoint 
subsets $A_{i}$ of $X$, where $i<\omega$, such that for every $n<\omega$, every $n$-uncountable Borel subset $B$ of $X^{n}$ and every $i<\omega$ we have

$$
\left|B \cap A_{i}^{n}\right|_{n}=2^{\omega} \text {. }
$$

Proof. Let us denote by $\mathscr{B}_{n}$ the family of all $n$-uncountable Borel subsets of $X^{n}$. Since there are at most continuum Borel subsets in a separable metric space, the cardinality of $\mathscr{B}_{n}$ is $\leqslant c$. Let $\left\{B_{\alpha}\right\}_{\alpha<c}$ be such an enumeration of all elements of $\mathscr{B}=\cup_{n=1}^{\infty} \Re_{n}$ that every element from $\mathscr{B}$ is listed continuum many times. For each $\alpha<c$ there exists exactly one $n(\alpha)$ such that $B_{\alpha} \in \mathscr{B}_{n(\alpha)}$.

For $\alpha<c$ and $i<\omega$ we will construct points $p(\alpha, i)$ belonging to $B_{\alpha}$ in such a way that

$$
\hat{p}(\alpha, i) \cap \hat{p}\left(\alpha^{\prime}, i^{\prime}\right)=\varnothing, \quad \text { if }(\alpha, i) \neq\left(\alpha^{\prime}, i^{\prime}\right) .
$$

Let $p(0, i), i<\omega$, be arbitrary points from $B_{0}$ such that $\hat{p}(0, i) \cap \hat{p}\left(0, i^{\prime}\right)=$ $\varnothing$, if $i \neq i^{\prime}$. Such points exist because $B_{0}$ is $n(0)$-uncountable. Let us take $\alpha<c$ and assume that we have already constructed points $p(\beta, i)$, for $\beta<\alpha$ and $i<\omega$. The set $Y=\bigcup\{\hat{p}(\beta, i): \beta<\alpha, i<\omega\}$ has cardinality less than $c$ and therefore by Theorem 1 the set

$$
B_{\alpha}^{*}=B_{\alpha} \backslash \bigcup_{j=1}^{n}\left(X^{j-1} \times Y \times X^{n-j}\right),
$$

where $n=n(\alpha)$, has $n$-cardinality continuum and consequently we can find for $i<\omega$ points $p(\alpha, i) \in B_{\alpha}^{*}$, such that $\hat{p}(\alpha, i) \cap \hat{p}\left(\alpha, i^{\prime}\right)=\varnothing$, if $i \neq i^{\prime}$ which completes the inductive construction. It is easy to see that (6) is satisfied.

Let us put $A_{i}=\cup_{\alpha<c}\{\hat{p}(\alpha, i)\}$. Clearly the sets $A_{i}, i<\omega$, are disjoint. If $n<\omega$ and $B$ is an $n$-uncountable Borel subset of $X^{n}$ then there exist continuum many ordinals $\alpha<c$ such that $B=B_{\alpha}$ and for every such $\alpha$ and every $i<\omega$ we have

$$
p(\alpha, i) \in B_{\alpha} \cap(\hat{p}(\alpha, i))^{n} \subset B \cap A_{i}^{n} .
$$

It follows from Lemma 1 and (6) that $\left|B \cap A_{i}{ }^{n}\right|_{n}=2^{\omega}$.

Corollary 4. Let $X$ be a separable complete metric space. There exists a subset $A$ of $X$ such that for every $n<\omega$, the complement of any Borel subset of $X^{n}$ containing either $A^{n}$ or $(X \backslash A)^{n}$ is n-countable.

Proof. Let $A_{i}$ 's be as in Theorem 2. Put $A=A_{0}$ and recall that the complement of a Borel set is a Borel set.

The following theorem can be proved in a similar way as Theorem 1 using the Theorem of Arhangel'skiǐ [A].

Theorem 3. Let $X$ be a first countable complete Lindelöf space. A closed subset $F$ of $X^{n}$ is either $n$-countable or has $n$-cardinality continuum.

Corollary 5. Let $X$ be a first countable compact space. A closed subset $F$ of $X^{n}$ is either $n$-countable or has $n$-cardinality continuum. 
COROLlaRY 6 (ČECH-PosPIŠIL-ARHANGEL'SKIĪ). A first countable compact space is either countable or has cardinality continuum.

REMARK 4. Theorem 3 can be generalized in the following way: Let $X$ be a first countable Hausdorff space. A complete Lindelöf subspace $A$ of $X^{n}$ is either $n$-countable or has $n$-cardinality continuum.

\section{REFERENCES}

[A] A. V. Arhangel'skii, The power of bicompacta with first axiom of cardinality, Dokl. Akad. Nauk SSSR 187 (1969), 967-968 = Soviet Math. Dokl. 10 (1969), 951-955.

[vD] E. van Douwen, A technique for constructing honest, locally compact submetrizable examples (to appear).

[E] R. Engelking, General topology, Polish Scientific Publishers, Warsaw, 1977.

[K] K. Kuratowski, Topology, vol. I, Academic Press, New York; PWN, Warsaw, 1966.

$\left[\mathbf{K}_{1}\right] \ldots$, Applications of the Baire-category method to the problem of independent sets, Fund. Math. 81 (1974), 65-72.

[P] T. C. Przymusiński, Normality and paracompactness in finite and countable cartesian products, Fund. Math. (to appear).

$\left[\mathbf{P}_{1}\right]$ _ On the dimension of product spaces and an example of $M$. Wage (to appear).

$\left[\mathbf{P}_{\mathbf{2}}\right]$ _ Products of perfectly normal spaces, Fund. Math. (to appear).

$\left[\mathbf{P}_{3}\right] \longrightarrow$, Topological properties of product spaces and the notion of $n$-cardinality, Topology Proceedings, vol. 2, 1977 (to appear).

Instytut Matematyczny PaN, Sniadeckich 8, 00-950 Warsaw, Poland 